\title{
DESARROLLO DE LA FORMACIÓN DEL PROFESORADO UNIVERSITARIO EN EL USO EDUCATIVO DE LA WEB SOCIAL: 20 CRITERIOS PARA SU DISEÑO E IMPLEMENTACIÓN
}

\author{
THE DEVELOPMENT OF THE FACULTY'S TRAINING IN THE \\ EDUCATIONAL USE OF THE SOCIAL WEB: 20 CRITERIA FOR HIS DESIGN \\ AND IMPLEMENTATION
}

\author{
Ana Mํ Rodera Bermúdez \\ arodera@uoc.edu \\ Universitat Oberta de Catalunya
}

\section{RESUMEN}

Este artículo presenta criterios para guiar el diseño y desarrollo de formaciones del profesorado universitario sobre el uso didáctico de la web 2.0. Los resultados proceden de un cuestionario aplicado a docentes y de una entrevista realizada a expertos en tecnología educativa. Se concluye que, la consideración de las necesidades de los docentes y la adopción de pedagogías abiertas, participativas, personalizadas y productivas promueven acciones formativas eficaces y actualizadas mediadas por las TIC.

PALABRAS CLAVE: Criterios de formación docente, uso educativo de la web 2.0, desarrollo profesional docente, web 2.0, profesorado universitario.

\section{ABSTRACT}

This paper presents criteria to guide the design and development of the faculty's training in the educational use of Web 2.0. The results come from a questionnaire applied to teachers and an interview experts in educational technology. In conclusion, consideration of: the teacher's needs and the adoption of an open, participatory, personalized and productive pedagogies mediated by the ICT, promotes effective and update training activities.

KEYWORDS: Criteria for faculty's training, educational use of the Web 2.0, professional development, Web 2.0, professors. 


\section{REVISIÓN Y NECESIDAD DE PUESTA A PUNTO DE LA FORMACIÓN DOCENTE EN EL USO DE LAS TIC}

Las transformaciones del modelo económico imperante, caracterizado por la revolución tecnológica y la globalización, están constituyéndose como fuentes de presión de cara a la generación de alteraciones en todos los niveles académicos del sistema educativo actual (Madrid, 2005).

Ante la situación anterior, diversos países están diseñando e implementando nuevos planes para la incorporación de las tecnologías de la información y la comunicación ( TIC $^{1}$ ) en la educación universitaria. Mediante dichos planes además de dotar de infraestructuras, se está realizando un especial hincapié en la formación del profesorado (UNESCO, 2008) con el fin de redefinir su papel en las aulas convirtiéndolos en agentes activos en el uso y aprovechamiento educativo de las web social (Casado, 2006).

Desde la universidad española, se han diseñado e implementado cinco modelos de formación docente (Zabalza, 2005) (1) basados en el apoyo mutuo, (2) centrados en programas de investigación-acción, (3) apoyados en el enriquecimiento doctrinal (por ejemplo, cursos y talleres convencionales, manejo de bibliografías especializadas), (4) que giran en torno a la reflexión y (5) fundamentados en la acreditación, que persiguen la consolidación de un cambio educativo y requieren la incorporación eficaz de las TIC.

Pero para poder integrar eficazmente las tecnologías en su práctica diaria, el docente tiene que sentirse seguro. Sin embargo, se evidencia que todavía las acciones formativas dirigidas al profesorado universitario carecen del impacto suficiente que permita aumentar la confianza del colectivo de profesores en el momento de utilizar los recursos de la web 2.0 en sus aulas (Gray, Thomas \& Lewis, 2010).

A su vez, se requiere empezar a concebir la formación del profesorado realmente como un aprendizaje a lo largo de toda la vida, orientada a la adquisición de competencias profesionalizadoras, entre las que se encuentran las competencias dirigidas a potenciar el desarrollo de aspectos metodológicos y de dominio de las tecnologías 2.0 como instrumentos al servicio de la acción docente (Imbernón, 2004).

Por consiguiente, parece imprescindible que cada universidad concrete y clarifique el perfil y demandas de sus profesores $y$, a partir de estos datos, diseñe la formación docente pertinente, con el fin de reducir su incertidumbre ante la situación actual de cambios constantes. Una vez realizado el proceso de concreción del perfil docente que cada universidad desea, se pueden tomar como base para el diseño de acciones los cuatro niveles de formación establecidos por Valcárcel (2003): "formación previa, inicial, continua y especializada en enseñanza disciplinar" (pp. 84-85).

En este sentido, este artículo pretende dar respuesta a ¿cómo deben plantearse las ofertas de formación permanente del profesorado universitario sobre el uso didáctico de la web social? Para ello se explicitan una serie de criterios que guíen el diseño e implementación de acciones de formación para los docentes universitarios eficaces, centradas en el uso educativo de las herramientas de la web 2.0 y potenciadoras del desarrollo profesional

\footnotetext{
${ }^{1}$ En este artículo se utilizan los términos TIC, web social, web 2.0, tecnologías 2.0 o tecnologías de la web social como sinónimos.
} 
docente $^{2}$.

\section{METODOLOGÍA DE LA INVESTIGACIÓN}

En este estudio se adoptó un paradigma interpretativo. La investigación efectuada dio lugar a una investigación centrada en el campo de la didáctica y en relación con las denominadas investigaciones en línea o e-researchs (Anderson \& Kanuka, 2003) debido tanto a la propia naturaleza de la temática abordada, como a los servicios web utilizados (para la implementación del cuestionario Limesurvey, http://www.limesurvey.org/es y para el desarrollo de la entrevista Skype, http://www.skype.com/intl/es/home/ combinado con MP3 Skype Recorder, http://voipcallrecording.com) a la hora de diseñar y aplicar los instrumentos de investigación.

La aproximación metodológica se basó en una metodología mixta, donde se administraron dos instrumentos de investigación:

1. Un cuestionario en línea, que fue aplicado a una población constituida por los profesores de la asignatura de NNTT aplicadas a la educación ( $\mathrm{N}=159)$, impartida en las especialidades de magisterio de las universidades públicas españolas. Se obtuvo una muestra representativa de participantes (35) que accedieron al cuestionario online y lo completaron. El servicio de software libre de realización de encuestas Limesurvey se usó a la hora de diseñar, distribuir y recoger las informaciones del cuestionario.

2. Una entrevista en línea, a 17 expertos en tecnología educativa del ámbito nacional e internacional. El servicio de comunicación Skype permitió realizar las entrevistas, que fueron grabadas mediante el programa MP3 Skype Recorder. Mediante la entrevista semiestructurada en línea se aportó en primer lugar, una visión profunda de la integración educativa del software social en la universidad a través de las percepciones expresadas por los informantes clave. En segundo lugar, favoreció la cristalización de criterios de mejora de la implementación educativa de las TIC en la universidad (Quivy \& Van Campenhoudt, 1997).

\section{Resultados del cuestionario en línea: formatos y contenidos de la formación recibida por el profesorado universitario}

El análisis efectuado sobre los datos obtenidos en el cuestionario en línea referidos a aspectos relacionados con la formación del profesorado universitario sobre el uso didáctico de las tecnologías de la web social arrojan informaciones acerca de: su participación o no en programas de formación, sus preferencias de formato a la hora de formarse y su particular opinión acerca de la distribución óptima de contenidos en las acciones formativas.

Tres cuartas partes de la muestra afirman haber participado en las formaciones sobre el uso didáctico de las herramientas de la web social (26 profesores, que representan un $74 \%$ de la muestra de estudio). La inexistencia de acciones formativas que respondan a sus

\footnotetext{
${ }^{2}$ Se entiende por desarrollo profesional, en sentido amplio, el desarrollo de una persona en su ámbito profesional. Más específicamente, se interpreta como el crecimiento profesional que alcanza un docente como resultado de su experiencia y examen sistemático de su quehacer didáctico (Glatthorn, 1995).
} 
necesidades particulares (20\%), por un lado y, la falta de tiempo (6\%), por otro, son las principales causas para no formarse.

En cuanto a la preferencia de modalidades de formación reglada, los docentes destacan, en primer lugar, los programas de formación para profesores o de actualización permanente (51\%); en segundo lugar, los congresos y conferencias (37\%); y en tercer lugar, los workshops, las jornadas y talleres (23\%).

La formación informal autodidacta basada en el uso de la web social es realizada por 32 de los docentes (92\%), compaginándola en todo momento con la formación de carácter reglado. Los profesores que se etiquetan como autodidactas señalan como las dos causas principales por las que han decidido autoformarse: la actualización profesional (29\%) y las inquietudes personales (26\%).

Otros argumentos relevantes a la hora de decantarse por una formación autodidacta son: la mejora que experimenta el profesorado de sus competencias profesionales (23\%), la transferencia de contenidos a su aula (23\%), la adecuada personalización de los contenidos (14\%), las soluciones que aportan a problemas profesionales habituales y la transferencia de los aprendizajes adquiridos al aula (14\%), y el ajuste de los contenidos a sus necesidades de desarrollo profesional (11\%).

En relación con la distribución óptima de carga de trabajo de los diferentes tipos de contenido en las formaciones sobre la utilización didáctica de los recursos 2.0, los docentes afirman que, debería priorizase, en primer lugar, el tratamiento de contenidos procedimentales vinculados con la implementación o experimentación directa en el aula de las tecnologías 2.0 como facilitadoras de los procesos de enseñanza-aprendizaje. Dichos contenidos deberían tener una carga del $50-60 \%$ del tiempo del curso. En segundo lugar, el desarrollo de contenidos de dominio técnico de las herramientas de la web social debería contar con una carga de trabajo del $20-30 \%$.

Por último, la profundización acerca de contenidos de carácter teórico relacionados con el significado de la cultura y pedagogía 2.0, cuenta con una carga para el profesorado del 10$20 \%$.

Se destaca en este apartado que: (1) casi un $75 \%$ del profesorado ha recibido algún tipo de formación, (2) los docentes prefieren formatos autodidactas (92\%) a programas reglados a la hora de formarse en el uso didáctico de la web 2.0, ya que les permiten actualizar sus conocimientos y responden a sus expectativas personales y (3) los contenidos procedimentales cobran un peso relevante en las formaciones (50-60\%) seguidos de los contenidos actitudinales y por último de los contenidos teóricos.

\section{Resultados de las entrevistas semiestructuradas en línea: Cambios de fondo y forma en las acciones formativas del profesorado universitario}

De las entrevistas semiestructuradas realizadas a los 17 expertos de tecnología educativa se desprenden los siguientes resultados que se han situado en dos grupos temáticos. El primero está centrado en la necesidad de modificar el diseño y desarrollo de los programas de formación docente en el uso didáctico de las TIC. Y el segundo está vinculado con el cambio en los contenidos formativos, de manera que promuevan habilidades didácticas y 
tecnológicas que ayuden a ir configurando los entornos personales de aprendizaje (PLE), así como las redes personales de aprendizaje (PLN) de los profesores universitarios.

Los entrevistados afirman que, se requiere una profunda revisión ${ }^{3}$ y mejora del diseño y desarrollo de las acciones formativas. Según los expertos en tecnología educativa, desde organismos educativos internacionales como el EEES o la UNESCO, también se está haciendo eco de la importancia de someter a valoración y modificación los programas de formación permanente del profesorado en materia de integración educativa de las TIC en educación superior. El sentido de esta reforma viene apoyado, por un lado, por la necesidad de establecer unas pautas realmente eficaces, adaptadas a las demandas del contexto actual y de los docentes. Por otro lado, al hecho de que los programas de formación en tecnología educativa vigentes resultan obsoletos, despersonalizados, descontextualizados y cerrados.

Para dar respuesta a la precaria situación de la formación del profesorado sobre la integración educativa de la web 2.0, los expertos entrevistados proponen implementar un proyecto formativo claro, actualizado y adaptado, de desarrollo profesional docente de acuerdo con estas nueve líneas rectoras:

1. Conocer las dimensiones sociales de la web 2.0. La web del siglo XXI se caracteriza por potenciar el carácter de consumidor-productor (prosumer), la compartición y la sabiduría de multitudes (wisdom of crowds).

2. Observar, revisar, analizar y repasar las herramientas de la web 2.0 a las que se pretende dar un uso didáctico, considerando los objetivos educativos perseguidos a la hora de seleccionarlas.

3. Promocionar acciones formativas que se centren de manera prioritaria en el trabajo de las competencias didácticas mediadas por las tecnologías de la web social, y no únicamente en aspectos técnicos. Mediante el desarrollo de estrategias que fomenten el trabajo en equipo y la motivación personal de los docentes.

4. Diseñar los planes formativos del profesorado contando con aquellos docentes innovadores, que ya están utilizando las TIC en sus aulas y que por tanto poseen experiencia en este ámbito.

5. Plantear una formación de abajo hacia arriba (bottom-up) es decir, a partir de la escucha de las necesidades del profesorado y trabajando contenidos que les permitan diseñar y aplicar acciones metodológicas mediadas por las TIC en sus aulas.

6. Reconocer al profesorado como aprendiz, experimentador, constructor y consultor. Para ello, se tiene que hacer uso de metodologías que fomenten el aprendizaje vicario, por medio de la observación y el intercambio de prácticas educativas exitosas mediadas por las TIC entre los profesores.

7. Desarrollar habilidades tecnológicas (o hard skills) en el profesorado a través de las acciones formativas ofertadas desde cada universidad. Se requiere una revisión continua y actualización de los recursos 2.0 en función de los objetivos y necesidades docentes. 
8. Organizar talleres de uso de las herramientas de la web social promoviendo la interactuación entre los participantes.

9. Ofrecer una capacitación básica a los docentes acerca del manejo del campus virtual propio de cada facultad.

Asimismo, los expertos afirman que los contenidos fundamentales que deben abordarse desde las propuestas formativas 2.0 para el profesorado pueden dividirse en dos grupos (Tabla 1). Un primer grupo centrado en la promoción del cambio de mentalidad y de un modelo pedagógico basado en la participación, la personalización y la producción. Y un segundo grupo centrado en el desarrollo del trabajo con servicios específicos referentes a la web social.

\section{Grupo 1: Contenidos relacionados con el cambio de mentalidad y de modelo pedagógico}

1. Clarificación de la concepción de web social y de sus posibilidades de uso educativo

2. Cambio del modelo pedagógico

3. Presentación de la generación del aprendizaje en forma de Red

4. Utilización de tecnologías que promuevan el "compartir" y el trabajo colaborativo a partir de la pertenencia a comunidades de práctica y a redes sociales educativas

5. Trabajo de la gestión de la información mediante servicios de sindicación y agregación (RSS) y del portfolio digital mediante la elaboración de blogs, wikis o espacios web que hacen de soporte del PLE del profesor.

\section{Grupo 2: Contenidos relacionados con el trabajo específico de servicios de la web} social

1. Realización de una buena distribución del tiempo a la hora de trabajar las herramientas de la web social, considerando desde un primer momento los diferentes niveles de conocimiento y uso, ritmos y estilos de aprendizaje vinculadas a las TIC por parte del profesorado.

2. Posesión de conocimiento suficiente acerca de cuestiones sobre la propiedad intelectual relacionadas con el uso de herramientas 2.0. Se requiere que el profesorado conozca las diferentes licencias Creative Commons, así como los sitios web donde es posible conseguirlas.

3. Explicitación de las funciones y del modo de empleo de las aplicaciones de la web social que hay en el mercado mediante la publicación de sencillos tutoriales.

Tabla 1. Contenidos formativos sobre el cambio de mentalidad, la pedagogía de aula y el trabajo de los servicios de la web social. Fuente: Elaboración propia.

Sobre la temática de la formación docente, los expertos mencionan el papel destacado, que en los últimos años, han desempeñado tanto los PLE como las PLN en el desarrollo, tanto personal como profesional, del profesorado. De este modo, los entrevistados consideran que las acciones formativas del profesorado tienen que diseñarse a partir de sus preferencias y necesidades. Es decir, promueven acciones del tipo bottom-up. 
En esta línea, los expertos consideran que es fundamental crear "ambientes de aprendizaje" que ayuden a promover: la explicitación y el desarrollo de aprendizajes personalizados a partir del trabajo colaborativo, la generación de redes de conocimiento vinculadas con la utilización de herramientas tecnológicas en el ámbito educativo, la potenciación del "aprender haciendo" (learning by doing), el aprendizaje abierto y el conocimiento compartido.

Según los entrevistados, el éxito de las propuestas formativas se corresponderá con la activación de un triple mecanismo de conexión que circulará a través de la sociedad, el mundo laboral y la universidad, y que debe promover el aprender desde la práctica o el aprender haciendo (learning by doing) considerando las particularidades de los contextos, del profesorado y de las tecnologías.

\section{DISCUSIÓN}

Al igual que apuntan Gutiérrez, Palacios y Torrego (2010), del análisis de los resultados expuestos se hace patente la necesidad de revisar y modificar la formación del profesorado universitario en activo con respecto a la integración y utilización de las TIC en sus aulas). Pero según Lara (2010) "falta capacidad para arriesgar y probar nuevas formas de interacción en procesos de aprendizaje donde profesores y alumnos producen y comparten conocimiento en modelos más horizontales. No es tanto un problema de disponibilidad de tecnología, como de cultura tecnológica y más aún, de cultura digital".

Coincidiendo con García-Valcárcel y Tejedor (2006), los resultados cuantitativos y cualitativos de este estudio evidencian la urgencia de replantearse las estrategias de formación que se vienen implementando a nivel de actualización y desarrollo profesional docente. Desde esta investigación se insiste especialmente en incidir sobre el diseño y puesta en práctica de pedagogías mediadas por las TIC en los contextos de aula, bajo planteamientos constructivistas y colaborativos más que en la presentación y consecución de un dominio técnico experto de múltiples servicios de la web social.

Los resultados presentados verifican que, las formaciones tienen que centrar su desarrollo, por un lado, en el trabajo de competencias didácticas y tecnológicas, tal y como también afirman Tejada y Giménez (2007) y Hernández y Quintero (2009). Además, estos últimos señalan que "la formación más demandada va más allá de la adquisición de conocimientos en el empleo de las TIC, requiriendo una formación más didáctica, estrechamente relacionada con sus necesidades y problemas reales a nivel de aula" (p. 116). Por otro lado, deben incentivar un proceso en espiral de evaluación y mejora constante.

Al respecto, Castañeda y Adell (2011) comentan que, la planificación de las acciones formativas debe centrarse en la búsqueda de posibilidades que permitan crear un espacio de aprendizaje que realmente responda a las necesidades formativas manifestadas por el profesorado. Se trata de establecer las bases que promuevan la emergencia de un entorno que permita mejorar la "pericia docente, entendida como "el constante e iterativo compromiso en la construcción y reconstrucción del conocimiento profesional utilizando varias perspectivas, incluyendo la investigación, con la intención de conceptualizar y resolver 
problemas" (Kelly, 2006, pág. 509), un entorno de aprendizaje enriquecido y personal desde el que desarrollarse como persona y profesional" (Castañeda y Adell, 2011, p. 6).

En esta línea, y coincidiendo con Oliveira, Cervera y Martí (2009) las informaciones recogidas en este trabajo apuntan a que la capacitación del profesorado en relación a las TIC debe adoptar un carácter disciplinar, pedagógico y tecnológico, basándose en el modelo teórico para el uso de la tecnología en educación Technological Pedagogical Content Knowledge Framework (TPACK) de Mishra y Koehler (2008).

El análisis de los datos referidos a las acciones informales de formación constata, como ya afirman Atwell y Hughes (2010) y Conole (2008) que, gracias a los procesos de autoformación desarrollados por los docentes en entornos virtuales y presenciales de aprendizaje, progresivamente se va configurando tanto su PLE como su PLN.

En definitiva, este estudio refrenda las conclusiones de Castañeda y Adell (2011) cuando aluden a que la generalización y evaluación continua de los PLEs así como, de las PLN tiene que convertirse en un elemento indisociable del proceso de aprendizaje y crecimiento de los docentes, tanto personal como profesional, a lo largo de toda la vida.

\section{CONCLUSIONES}

A la hora de dar respuesta al cómo deben plantearse las ofertas de formación permanente del profesorado universitario sobre el uso didáctico de la web social, en primer lugar resulta fundamental que dichas ofertas formativas se entiendan como soluciones que ofrecen respuestas concretas a las necesidades intrínsecas de los docentes.

En segundo lugar, es imprescindible que los programas formativos reglados ayuden a mejorar los procesos de enseñanza-aprendizaje, promuevan el reconocimiento entre el profesorado (que afecta positivamente a su DPD), respondan a cuestiones referidas al significado y consecuencias que tiene educar, favorezcan el conocimiento, y permitan que los docentes pierdan progresivamente el miedo a experimentar en las aulas con pedagogías mediadas por los servicios disponibles en la web social.

En tercer lugar, la formación debe dirigirse hacia la consolidación de ambientes de aprendizaje personalizados y colaborativos, donde se otorgue prioridad a los aprendizajes experimentales (learning by doing).

En cuarto lugar, desde este trabajo se aboga porque, en el planteamiento de la formación se contemplen estos ocho objetivos:

1. Adoptar una perspectiva totalmente constructivista y conectivista, en las fases de diseño, desarrollo y evaluación del desarrollo de la propuesta formativa.

2. Permitir la adaptación de la formación a las particularidades de cada contexto, docente, alumnado y tecnología, a partir del establecimiento de formaciones que partan de una perspectiva bottom up y de las expectativas y necesidades sentidas por los agentes educativos.

3. Favorecer el conocimiento de la web social y la cultura 2.0 ya que, ambas constituyen variables que inciden positivamente sobre el conocimiento de uso 
educativo de las TIC en las aulas.

4. Revisar y actualizar los objetivos de aprendizaje de los planes de formación y los diferentes tipos de herramientas 2.0

5. Priorizar y ofrecer una capacitación avanzada al profesorado, tanto a nivel didáctico como tecnológico, acerca del manejo del campus virtual y del LMS de su facultad así como, de diversos recursos de la web social, que le permita mejorar su DPD.

6. Reconocer al docente como un aprendiz, constructor y facilitador de los procesos de enseñanza-aprendizaje, favoreciendo su participación en el cambio de su propia práctica profesional mediada por las TIC.

7. Potenciar la transferencia de los aprendizajes y contenidos de la formación al espacio del aula de los docentes, ajustando los contenidos, didácticos y tecnológicos, a las necesidades concretas manifestadas por el profesorado.

8. Planificar y llevar a la práctica pequeños talleres acerca del uso educativo de un determinado servicio de la web social en contextos educativos diversos, a través de tareas experimentales, reales y basadas en proyectos.

En quinto lugar, también desde estas líneas se insta a que, los contenidos planificados en las formaciones se centren en el fomento del cambio de mentalidad, tendente hacia la asimilación de la cultura y del modelo pedagógico 2.0. Se apuesta por trabajar con el objetivo de consensuar pedagogías personalizadas, participativas y productivas que favorezcan la adquisición de conocimientos en las aulas. Además, se tiene que profundizar acerca del funcionamiento técnico de determinados tipos de herramientas de la web social.

En sexto lugar, los planes de formación deben favorecer el trabajo tanto de las hardskills como de las softskills porque, un alto dominio de ambas implicará que los docentes ejerzan roles innovadores. La adopción de estos roles dará lugar a que los profesores actúen como facilitadores de los procesos de aprendizaje, suscitando el papel activo del alumno en la construcción de su propio conocimiento y llevando a cabo un máximo aprovechamiento de las TIC en los procesos de enseñanza-aprendizaje.

En séptimo lugar, en cuanto a los contenidos que se deben seguir en la implementación de un dominio didáctico y técnico adecuado de los servicios de la web social, las informaciones obtenidas dan pie a afirmar que, estos contenidos (principalmente procedimentales) tienen que trabajarse junto con el desarrollo de las competencias didácticas del profesorado.

En octavo lugar, se identifica en este estudio que, la óptima combinación y trabajo de contenidos didácticos y tecnológicos permitirá desarrolla PLNs y PLEs eficaces en las formaciones de los docentes.

En noveno lugar, es importante que las formaciones se anticipen a las necesidades de los profesores, ayudándoles a resolver las dificultades inmediatas que les vayan surgiendo, de manera que no se sientan solos durante el proceso de práctica didáctica de las tecnologías 2.0 .

Por último, desde este trabajo se constata que, la formación docente vinculada a una integración de calidad de las TIC en las aulas universitarias cobrará valor en la medida en que consiga establecer una conexión real entre los objetivos, los contenidos y los contextos 
social, laboral y educativo.

Como colofón final de este artículo se presentan 20 criterios susceptibles de ser aplicados en el diseño y desarrollo de futuras formaciones docentes del profesorado universitario centradas en maximizar la eficacia educativa de la web social.

1. Apuesta por una formación abierta, promotora de aprendizajes de calidad y transferibles al aula, diseñada de manera bottom up, no tecnocéntrica, ni teórica, interactiva y participativa, basada en los conocimientos previos y en las necesidades sentidas de los docentes, en modelos de relación horizontales, pedagogías 2.0 y en didácticas constructivistas, conectivistas y colaborativas y mediadas por las tecnologías.

2. Consideración del modelo TPACK como posible punto de partida de cara al diseño y desarrollo de las acciones formativas del profesorado.

3. Implementación de formaciones que favorezcan la conciliación entre los tiempos de formación y de actuación profesional.

4. Inclusión en el diseño de la formación tanto de expertos en tecnología educativa como de docentes con experiencia en el uso didáctico de las tecnologías.

5. Promoción del conocimiento compartido a través de la organización de jornadas, congresos o de la utilización de las tecnologías 2.0 (como por ejemplo las redes sociales o las redes sociales educativas) con el fin compartir experiencias, dudas y de trabajar los PLEs y las PLNs del profesorado.

6. Diseño y desarrollo de planes de formación docente o de desarrollo profesional docente favorecedores del cambio de mentalidad hacia la cultura 2.0 en las aulas, la correcta alfabetización digital y el dominio tecnológico de las TIC.

7. Análisis y reflexión del contexto social, laboral y universitario en el momento de elaborar de acciones formativas para el profesorado.

8. Establecimiento de mecanismos de acreditación de las formaciones desarrolladas por el profesorado en contextos virtuales o presenciales informales.

9. Fomento del trabajo de los servicios relacionados con el almacenamiento y recepción de información en las formaciones.

10. Organización de jornadas formativas (como por ejemplo los Educamps) donde se parta de las necesidades del profesorado y donde este último se constituya como el auténtico protagonista del evento y la tecnología restará al servicio de la mejora educativa.

11. Activación de acciones en las formaciones docentes encaminadas a: formar al profesorado en el uso didáctico y tecnológico de las herramientas de la web social, adoptar diferentes estilos de facilitación, presentación y discusión, determinar tiempos flexibles, crear espacios de comunicación y trabajo debidamente acondicionados, promocionar tareas de inmersión en el uso educativo de la web social por parte del profesorado universitario, etc.

12. Trabajo de dos tipos de contenidos prioritarios: el cambio de actitud y metodológico y el dominio técnico de las herramientas de la web social.

13. Distribución óptima de los porcentajes de trabajo de las diversas temáticas: $50-60 \%$ contenidos prácticos relacionados con el uso educativo del software social, $20-30 \%$ contenidos de dominio técnico y $10-20 \%$ contenidos teóricos.

14. Planteamiento de actividades en las formaciones encaminadas a favorecer el 
autorreconocimiento, la socialización, la evidencia de habilidades, la interacción entre los participantes y las habilidades metodológicas y técnicas.

15. Generación de productos internos y externos derivados de la formación. Los primeros sirven para agilizar el trabajo de los organizadores y los segundos para difundir el evento (backchannels).

16. Implicación de participantes y organizadores en una fase de evaluación y mejora de la formación, a través de la aplicación de cuestionarios, entrevistas, etc.

17. Realización de una valoración extensiva de los aprendizajes tras la participación en la formación, con el fin de averiguar el grado de aplicación y las dificultades existentes.

18. Consideración de las posibles transferencias de los aprendizajes acerca del uso educativo de los servicios web adquiridos en las aulas, en el momento de diseñar una acción formativa para los docentes.

19. Trabajo en las actividades formativas de las siguientes competencias: promoción de su actualización en cuanto a la aplicación didáctica de las TIC, desarrollo de su PLE y formación metodológica y tecnológica (alfabetización tecnológica).

20. Asociación del PLE al trabajo de una cultura abierta e innovadora, que fomente la construcción de PLNs entre los docentes.

Tabla 2. Criterios para la integración educativa de las TIC en la Dimensión de formación docente

Fuente: Elaboración propia

Estos criterios afectan a todas aquellas actividades que permiten reflexionar, diseñar y adaptar la formación continua del profesorado "en", "sobre" y "con" las tecnologías de la web 2.0. Su puesta en práctica en la configuración e implementación de acciones formativas regladas y su posterior valoración permitirá verificar su eficacia, así como la necesidad de ser redefinidos, ampliados o eliminados.

\section{REFERENCIAS}

ANDERSON, T., \& KANUKA, H. (2003). E-research: methods, strategies, and issues - Terry Anderson, Heather Kanuka - Google Libros. Recuperado de http://books.google.es/books?id=OiF8QgAACAAJ\&dq=eresearch+anderson+and+kanuka\&hl=es\&sa=X\&ei=Ind9T6bGNqKy0QWAvK2zDQ\&ved=0CDM Q6AEWAA

AREA, M. (2006). Veinte años de políticas institucionales para incorporar las tecnologías de la información y comunicación al sistema escolar. En J.Mạ. Sancho, (Coord.), Tecnologías para transformar la educación. Madrid: Akal.

ATTWELL, G., \& HUGHES, J. (2010). Pedagogic Approaches to Using Technology for Learning. Recuperado de http://es.scribd.com/doc/56715291/Pedagogical-Appraches-for-UsingTechnology-Literature-Review-January-11-FINAL-1

CASADO, R. (2006). Convergencia con Europa y cambio en la universidad. Los profesores y las nuevas tecnologías como elementos clave en el nuevo modelo de aprendizaje del Espacio 
Europeo de Educación Superior. EDUTEC. Revista Electrónica de Tecnología Educativa, (20). Recuperado de http://www.uib.es/depart/gte/gte/edutec-e/revelec20/casado20.htm

CASTAÑEDA, L. y ADELL, J. (2011). El desarrollo profesional de los docentes en entornos personales de aprendizaje (PLE). En R. Roig, R. \& C. Laneve, (Eds.). La práctica educativa en la sociedad de la información: Innovación a través de la investigación. Alcoy: Marfil. Recuperado

de http://digitum.um.es/xmlui/bitstream/10201/24647/1/CastanedaAdell2011preprint.pdf

CONOLE, G. (2008). New Schemas for Mapping Pedagogies and Technologies. Ariadne, 56. Recuperado de http://www.ariadne.ac.uk/issue56/conole/

GARCÍA-VALCÁRCEL, A. y TEJEDOR, F.J. (2006). Condicionantes (actitudes, conocimientos, usos, intereses, necesidades formativas) a tener en cuenta en la formación del profesado no universitario en TIC. Revista Enseñanza. Anuario Interuniversitario de Didáctica, 23, 115-14. Recuperado http://gredos.usal.es/ispui/bitstream/10366/18421/1/DDOMI Condicionantesactitudes.pdf

GLATTHORN, A. (1995). Teacher Development. En Anderson, L. (Ed.). International enciclopedy of teaching and teacher education. Londres: Pergamon Press.

GRAY, L., THOMAS, N., \& LEWIS, L. (2010). Teachers' Use of Educational Technology in U.S. Washington, DC. Public Schools: 2009 (NCES 2010-040).

GUTIÉRREZ, A., PALACIOS, A. y TORREGO, I. (2010). La formación de los futuros maestros y la integración de las TIC en la educación: anatomía de un desencuentro. Recuperado de http://www.oei.es/noticias/spip.php?article7364

IMBERNÓN, F. (2004). La profesión docente desde el punto de vista internacional ¿qué dicen los informes? Revista de Educación (340), 41-49.

KAPLOWITZ, M.D., HADLOCK, T.D. \& LEVINE, R. (2004). A comparison of Weand mail survey response rates. En Public Opinion Quarterly 68. Spring. 94-101.

LARA, T. (2010). Entrevista a Tíscar Lara. Mosaic. Tecnologías y Comunicación Multimedia. Recuperado de http://mosaic.uoc.edu/2010/07/16/tiscar-lara/

MADRID, J. M. (2005). La formación y la evaluación docente del profesorado universitario ante el espacio europeo de educación superior. Educatio, (23). Recuperado de http://revistas.um.es/index.php/educatio/article/viewFile/117/101

MISHRA, P., \& KOEHLER, M. (2008). Introducing Technologial Pedagogical Content Knowledge. Presentado en Annual Meeting of the American Research Association AERA, New York. Recuperado de http://www.wiziq.com/tutorial/71616-Mishra-amp-Koehler-2008

OLIVEIRA, J. CERVERA, M. y MARTÍ, M. (2009). Learning as representation and respresentation as learning. A theoretical framework for teacher knowledge in the digital age. Proceedings of World Conference on Educational Multimedia, Hypermedia and Telecommunications. VA: AACE. 
QUIVY, R. \& VAN CAMPENHOUDT, L. (1997). Manual de recerca en ciències socials. Barcelona: Herder.

SANGRÀ, A. y GONZÁLEZ, M. (2004). La transformación de las universidades a través de las TIC. Discursos y prácticas. Barcelona: Editorial UOC.

TEJADA, J. y GIMÉNEZ, V. (COORDS.) (2007). Formación de formadores. Escenario Institucional. Volumen 2. Madrid: Thomson-Paraninfo.

UNESCO. (2008). Estándares de competencia en TIC para docentes. Recuperado de http://www.eduteka.org/EstandaresDocentesUnesco.php

VALCÁRCEL, M. (2003). La preparación del profesorado universitario español para la Convergencia Europea en Educación Superior. Córdoba: Universidad de Córdoba.

ZABALZA, M. A. (2005). La docencia en el nuevo marco de las enseñanzas universitarias. En C. Ruiz-Rivas, (Ed.). Curso Profesorado y Políticas Universitarias de Calidad. Santander: UIMP.

Para citar este artículo:

Rodera, A. M. (2013). Desarrollo de la formación del profesorado universitario en el uso educativo de la web social: 20 criterios para su diseño e implementación. EDUTEC, Revista Electrónica de Tecnología Educativa, 45.4 Recuperado el $\mathrm{dd} / \mathrm{mm} / \mathrm{aa}$ de http://edutec.rediris.es/Revelec2/Revelec45/formacion prfesorado universitario uso educativo w eb social creterios.html 